\title{
LAS REGLAS DE LA VIDA, LAUDATO SI', Y TODOS NOSOTROS
}

\author{
JAMES A. F. STONER
}

Facultad de Negocios Gabelli

Universidad de Fordham

Nueva York, Nueva York, EE. UU. stoner@fordham.edu

En The Serengeti Rules: The quest to discover how life works and why it matters ${ }^{1}$ (Las reglas del Serengueti: La búsqueda para descubrir cómo funciona la vida y por qué vale la pena), Sean B. Carroll explora cómo los investigadores pioneros investigaron preguntas como estas: “¿Cómo funciona la vida?" y “¿Cómo hace la naturaleza para producir la cantidad correcta de cebras y leones en la sabana africana, o de peces en el océano?". Y el por qué las respuestas que encontraron a las preguntas como estas, son importantes para nosotros en el presente mientras luchamos contra algunos de nuestros problemas actuales-especialmente los que tienen que ver con la insostenibilidad del planeta.

En el último capítulo, titulado "Después: Las Reglas de la Vida," Carroll escribe: "Yo afirmé al inicio de este libro que nuestro incremento en la maestría de la biología era un gran catalizador de los cambios dramáticos en la cantidad y cualidad de la vida humana en el último siglo." Después él se pregunta, “Entonces qué papel tendrá la biología en los asuntos humanos en el próximo siglo?"

Después de un corto comentario del sumario sobre las contribuciones pasadas de la biología a nuestro bienestar, al igual que al de las otras especies que él ha explorado en todo su libro y notando posibles futuras contribuciones, él escribe:

${ }^{1}$ Los fragmentos tomados de The Serengeti Rules son citados con el permiso de Sean B. Carroll y su casa editorial, Princeton University Press, lo cual agradecemos. 
Pero, aunque por más deseable que sean estos avances, no son los desafíos más trascendentales para los biólogos y la sociedad. Al contrario, es el decadente estado de salud de nuestro hogar y lo que eso significa para la capacidad de los ecosistemas de la tierra de sostener vida, mucho menos otras criaturas. Hemos creado una situación ecológicamente extraordinaria en la cual somos el depredador principal y el mayor consumidor en todos los hábitats. Como advierte Robert Paine, "Los humanos ciertamente son los actores predominantes y serán los perdedores principales si las reglas no son aceptadas y los ecosistemas globales continúan a deteriorarse." Ahora mismo la única especie que puede regularnos somos nosotros mismos.

Si el lema para el siglo 20 fue "Vivir Mejor por medio de la Medicina," el lema para el siglo 21 debe ser "Vivir Mejor por medio de la Ecología." Afortunadamente, no son solo los científicos quienes están profundamente preocupados o quienes reconocen la primacía de la ecología. Te podrás sorprender al enterarte quien fue el que dijo lo siguiente (ciertamente yo lo estuve):

Frecuentemente, cuando ciertas especies son explotadas comercialmente, poca atención es puesta a sus ciclos reproductivos para poder prevenir su reducción y el consiguiente desbalance del ecosistema.... Cuidar a los ecosistemas requiere una visión a largo plazo.... Cuando ciertas especies son destruidas o seriamente vulneras, los costos generados son incalculables. Podemos ser testigos silenciosos a injusticias terribles si pensamos que podemos obtener grandes beneficios al hacer que el resto de la humanidad, presente y futura page un costo inimaginablemente alto por la deterioración ambiental.

El autor es el Papa Francisco, quien publicó una Carta Encíclica extraordinaria sobre el medio ambiente en junio del 2015 "Sobre el cuidado de nuestro hogar compartido." Las encíclicas usualmente son utilizadas para dirigirse a la dirigencia Católica sobre temas importantes de doctrina. En esta instancia excepcional, el pontífice expresó su deseo "de dirigirse a todas las personas que viven en este planeta" y "para entrar en un dialogo con todas las personas sobre nuestro hogar compartido."

El documento de 183 páginas es impresionante por su alcance ecológico y por su franqueza. Después de analizar sistemáticamente los diferentes tipos de degradación, incluyendo la contaminación y el cambio climático, la contaminación del agua, y la pérdida de biodiversidad y la extinción de las especies, y los actos humanos que las han causado, el Papa Francisco dice:

Solo necesitamos mirar con franqueza los hechos y ver que nuestro hogar compartido está cayendo en una deseperación real.... Nosotros podemos ver las señales de que las cosas están llegando a su punto de quiebre, causado por el apresuramiento del cambio y la degradación. 
El Papa definitivamente coincide con la percepción de que, si vamos a llegar al próximo siglo con suficiente agua, tierras y mares productivos, y la comida para sostener a quizás 10 mil millones de personas, y al mismo tiempo preservar la vida de las criaturas, las reglas de la ecología tendrán que convertirse en las reglas de la vida. Por ende, él hace un llamado global para tener una "discusión ecológica," usando ese término en connotaciones espirituales y del diario vivir para un cambio profundo de corazón y mentalidad. (Carroll, 2016: 203-204)

El llamado explícito del Papa Francisco por un dialogo sobre la necesidad de una discusión sobre la ecología y su llamado delicadamente redactado pidiendo actos que protejan y nutran a nuestro hogar compartido han sido replicados en muchos lugares, incluidas las páginas de esta revista en su edición enfocada en Laudato si' (Vol. 5, No. 1), editadas por nuestro editor invitado Allen Tropea-Gray. Los llamados del Papa Francisco también han contribuyo a la resolución aprobada el 18 de Julio del 2016, en el encuentro conjunto del Foro Mundial de Asociación Internacional de Facultades de Negocios Jesuitas (World Forum of the International Association of Jesuit Business Schools (IAJBS)) y el Encuentro Anual de los Colegios Jesuitas de Educación Empresarial (Annual Meeting of the Colleagues in Jesuit Business Education (CJBE)) en Nairobi, Kenia:

El encuentro anual de la IAJBS solicita que los dirigentes de la IAJBS, los dirigentes de CJBE, y el resto de la red Jesuita de Facultades de Negocios trabajen unidos para aplicar al concurso 100\&Change de la Fundación MacArthur por 100 millones de dólares con un proyecto para transformar la educación empresarial Jesuita para que esté completamente alineada con la sabiduría de Laudato $\mathrm{Si}^{\prime}$, con nuestros principios educativos universales Jesuitas, y con la necesidad de una sostenibilidad global, justicia social, y la reducción de la pobreza.

Los artículos en esta edición de la revista continúan en este hilo. Los escritos de Kent Fairfield, Carl Obermiller \& Matt Isaac, y Maggie Eusebio se expresan de una manera directa sobre las preocupaciones expresadas por el Papa Francisco y en las oportunidades existentes para tomar acciones constructivas para responder a ellas; los de Michael Urick, Muyang Li, Selin Konur, \& Terrance Smith y Marinilka B. Kimbro, Ajay T. Abraham, C. Jay Lambe, \& Victoria Jones también nos aportan guías de cómo podemos tomar el tipo de acciones que nos ayudaran a luchar contra los problemas que hemos creado para nosotros mismo, y para los hijos de nuestros hijos.

Construir una manera de vivir en el mundo con una mentalidad de sostenibilidad es un paso fundamental—quizás el más importante— 
para cada uno de nosotros. En "Educando por una Mentalidad de Sostenibilidad," Kent Fairfield elabora sobre sus experiencias didácticas al tratar de enfrentar el problema de ayudar a sus estudiantes y otros a adquirir el conocimiento, las perspectivas, y los compromisos necesarios para la "conversión ecológica" que el Papa Francisco y otros han pedido. Él observa que cada vez más, los jóvenes profesionales bien preparados requieren ser expuestos y obtener un pesanmiento crítico a los problemas ambientales, sociales, políticos y empresariales, sea que esto solo sea necesario como contexto para el trabajo o como parte central de sus responsabilidades.

Reflexionando sobre su trabajo en una facultad de negocios, él nota que la competencia básica actual requiere conocimientos sobre el cambio climático, pensamiento de sistemas, y problemas humanitarios. Los graduandos deben tener familiaridad con los desafíos desconcertadores de los negocios y también con lo que están haciendo las compañías sostenibles ahora. En efecto, Fairfield argumenta que transmitir el aprendizaje cognitivo es solo el punto de partida para los profesores, mientras que adquirirlo es solo la base para un mayor crecimiento por parte de los estudiantes. Los verdaderamente educados han obtenido un aprendizaje experimental de fondo que informa de una nueva visión mundial. Él siente que la meta más trascendental a la que debe aspirar un profesor es de permitir que sus estudiantes empiecen a adquirir una nueva mentalidad o una nueva "manera de ser," aceptando sus sentimientos, su propósito de vida, y su identidad. Su artículo sugiere maneras en las que todos nosotros podemos trabajar hacia esa meta-en nuestro salón y más allá.

El incremento de concientización que tienen las mujeres sobre nuestra precaria situación ecológica y su mayor compromiso al tipo de dialogo pedido por Francisco ha sido evidente para muchos por varios años. En “¿Son los Hombres Verdes de Venus?," Carl Obermiller y Matt Isaac elaboran sobre el hallazgo consistente de la investigación sostenible que indica que las mujeres son más amigas de lo ecológico que los hombres, una brecha usualmente asociada a las diferencias de socialización. La investigación que ellos utilizan explora un proceso corolario-la asociación cognitiva del ambientalismo con la feminidad y las consiguientes respuestas negativas de los hombres basadas en sus esfuerzos de proteger su identidad masculina. En dos estudios, ellos replican los hallazgos recientes (Brough, Wilkie, Ma, Isaac, \& Gal, 2016) de una asociación mental entre el ambientalismo y la feminidad (de ambos mujeres y hombres) y la consiguiente reducción en la efectividad de los llamados ambientales convencionales para los hombres. 
Mas allá de documentar los posibles efectos de la asociación verde-femenina implícita sobre los juicios explícitos y las intenciones de comportamiento de los hombres, ellos también investigaron dos métodos para superar las asociaciones implícitas. En su primer estudio, ellos examinan si publicitar escepticismo y/o conocimientos sobre sostenibilidad podría moderar las consecuencias de la asociación verdefemenina. Sus hallazgos fueron que los participantes que tuvieron mayor publicidad escéptica eran menos propensos a exhibir la asociación verde-femenina es sus juicios explícitos. Sin embargo, ninguno de los estudios encontró evidencia de que aumento sobre el conocimiento de la sostenibilidad eliminaba o disminuía las posibles consecuencias de una asociación verde-femenina, aunque el segundo estudio demostró efectos generalmente positivos de un aumento en conocimiento de la sostenibilidad sobre las respuestas a un llamado ambiental.

Obermiller y Isaac también exploraron la posibilidad de contrarrestar la asociación verde-mujer implícita al crear un llamado ambiental con elementos de posicionamiento de marca distintivamente masculinos. Dos versiones de un llamado ambiental fueron producidas, uno con elementos de marca masculinos y el otro con femeninos. Mientras los hombres y las mujeres reaccionario de la misma manera al posicionamiento de marca masculino, la mayoría de las respuestas positivas al posicionamiento femenino fueron las mujeres.

Los autores notan que a pesar de que un aumento en el conocimiento de sostenibilidad parece resultar generalmente en un comportamiento más proambiental, no parece moderar los posibles efectos de la asociación implícita verde-femenina. Aun así, ellos son optimistas al final de su proyecto de que una mayor cantidad de publicidad escéptica puede ayudar a reducir el impacto de la asociación verde-femenina, y que el aumento del conocimiento de sostenibilidad puede reducir las asociaciones negativas del ambientalismo para los hombres al largo plazo.

Se ha dicho en una manera no trivial que "la insostenibilidad global es un problema espiritual." En "La Espiritualidad y la Sostenibilidad en los Negocios: El Caso de Las Fincas de Café en Amadeo, Cavite," Maggie Eusebio enfatiza la estrecha conexión entre la espiritualidad y la conversión ecológica. De la misma manera que el Papa Francisco indica en Laudato si', ella nota que una conversión espiritual interna es necesaria para generar un comportamiento responsable que pueda contribuir en la curación de nuestro hogar compartido-un hogar que llora por la violencia cometida en su contra. Ella habla de las tierras de cultivo de Amadeo, Cavite como "nuestra Hermana, la Tierra Madre," e insta que es tiempo de ayudarla, y que esto se puede lograr por medio de una conversión ecológica y espiritual. 
Para apoyar su convicción que tal conversión contribuirá a curar a nuestro hogar compartido, Eusebio reporta que su investigación encontró que la sostenibilidad de las fincas de café en Amadeo esta positivamente asociada con la espiritualidad intrínseca de los dueños, denominado por ella como una mayordomía Dios-céntrica de las tierras de cultivo, conlleva a fincas más sostenibles. Ella sugiere que los hallazgos de su investigación pueden ser extendidos a otros tipos de negocios, proponiendo que una mayordomía Dios-céntrica de los negocios llevaría a más negocios sostenibles.

El propósito de la "resolución de Nairobi" notado anteriormente, no es solo el de transformar la educación de negocios Jesuita para su propio bien; al contrario, es para usar la reforma de toda la educación de negocios para transformar el sistema de producción, distribución, y consumo de los bienes y servicios que necesita nuestra comunidad global-y en particular, transformar como las organizaciones empresariales ejercen su papel en la sociedad. Michael Urick, Muyang Li, Selin Konur, y Terrance Smith se enfocan en la necesidad de transformar estas prácticas de producción globales. Ellos elaboran sobre los paralelos en los que hemos estado aprendiendo durante las últimas siete décadas, en nuestros intentos de integrar en nuestras organizaciones la revolución de calidad inspirada por W. Edwards Deming, Joseph M. Juran, Kaoru Ishikawa, y muchos más, y el tipo de transformación mucho más profunda que necesitaremos al avanzar, si somos capaces de crear un sistema de producción-distribución-consumo consistente con un mundo "floreciente" (Ehrenfeld, 2010).

Urick et al. observan que las culturas enfocadas en excelencia operacional (EO), la cual permite un mejoramiento continuo, reducción de desechos, y de resolución de problemas, puede ayudar a organizaciones (y sociedades) a alcanzar una mayor sostenibilidad. Ciertamente, muchos investigadores en estudios anteriores se han enfocado en traer las "herramientas" de EO al interior de las organizaciones y convertirlas en parte de sus culturas, pero muchas organizaciones han reportado desafíos en su intento de implementar este tipo de cultura.

Urick y sus colegas argumentan vehementemente en contra de enfoques reducidos puramente a los desafíos técnicos de integrar practicas sostenibles consistentes en las organizaciones. Ellos observan que las investigaciones sobre los cambios culturales sugieren que los aspectos sociales en una organización suelen presentar mayores desafíos que los técnicos. Usando el enfoque de la teoría fundamentada para explorar los aspectos sociales de las culturas EO, ellos determinan que los aspectos sociales del trabajo representan mayores obstáculos para implementar mejorías continuas e iniciativas de excelencia operacional. Podemos 
asumir por extensión entonces que los aspectos sociales tienen una alta probabilidad, quizás más alta, de poner obstáculos para transformaciones hacia prácticas empresariales sostenibles.

Los autores también reportan que los obstáculos sociales para la implementación de EO pueden surgir de factores interpersonales tales como problemas de comunicación, el rechazo de algunos colegas de aceptar el cambio, y relaciones en el lugar de trabajo. Al igual que los factores organizacionales, como trato a los empleados (incluyendo nivel de responsabilidad y empoderamiento), valores culturales propios de la organización, y características formales organizacionales (incluyendo títulos formales, compromiso de la jefatura, tamaño del grupo, y educación/entrenamiento).

Por ende, Urick et al. enfatizan la importancia de reconocer las barreras sociales y de encontrar caminos sociales para descubrir cómo "escalar el Monte Sostenibilidad," usando el termino usado por Ray Anderson para describir la meta de Interface de convertirse en una compañía completamente alineada con las necesidades de un mundo sostenible (Fundación Ray Anderson, n.d.).

En "Responsabilidad Social Empresarial: La Eficacia de Alianzas Emparejadas entre Organizaciones Sin Ánimo de Lucro y Multinacionales en los Mercados Emergentes y en Desarrollo," Marinilka B. Kimbro, Ajay T. Abraham, C. Jay Lambe, y Victoria Jones se enfocan en cómo las empresas multinacionales (MNEs por sus siglas en inglés) pueden incrementar la efectividad de su sostenibilidad global e iniciativas de Responsabilidad Social Empresarial (RSE). Reconociendo la importancia de esfuerzos de RSE bien diseñados y cubrimiento positivo de los medios para lograr y mantener programas efectivos de RSE y sostenibilidad, ellos investigan las respuestas del mercado a las iniciativas de RSE cuando las MNEs y las organizaciones sin ánimo de lucro (OSAL) forman alianzas "emparejadas." Ellos discuten tres iniciativas de esa índole, formadas por MNEs y OSALs locales con un buen "emparejamiento" de objetivos comunes y capacidades complementarias: La Asistencia de Katrina de Walmart, Conexión de Campo de Infosys, y el Proyecto Shakti de Unilever.

Kimbro et al. miden la efectividad de estas actividades RSE por medio de un análisis de serie temporal de intensidad mediática. Los resultados indican un fuerte incremento en la cobertura mediática alrededor de eventos de RSE de estas tres MNE/OSAL alianzas emparejadas, y también a una respuesta positiva más fuerte cuando la MNE opera en un ámbito local en vez de uno extranjero. Además, este efecto positivo es más alto en mercados emergentes que en mercados desarrollados. 
Motivados por el deseo de responder a la creciente literatura que argumenta que los consumidores reaccionan a las iniciativas de RSE con un escepticismo paulatino, los autores postulan dos explicaciones generales para explicar la falta de reacciones positivas a las actividades de RSE: atribuciones negativas del mercado, la cual suele dominar la literatura, y una explicación menos explorada de procesos empresariales. Las atribuciones negativas del mercado nacen de una percepción creciente que las actividades de RSE son egocéntricas y que les falta legitimidad porque las compañías se pueden aprovechar y usar la RSE como un mecanismo de "ecoblanqueamiento" (greenwashing). En efecto, esta visión es compartida por el Papa Francisco en Laudato si' (p. 40): "hay demasiados intereses privados, y los intereses económicos terminan triunfando sobre el bien común y manipulando la información para que sus planes no se vean afectados."

La falta de respuestas positivas a la RSE y las actividades de sostenibilidad también pueden ser atribuidas a procesos operaciones inefectivos. Estas respuestas negativas pueden surgir de réplicas desinformadas de métodos operacionales basados en el mercado para desarrollar actividades RSE que ignoran que los actores en los contextos de RSE son muy diferentes a los clientes de sus negocios principales. Los mismos procesos inefectivos también pueden resultar de la falta de entendimiento local, cultural, situacional necesarios para planificar y ejecutar efectivamente iniciativas de RSE y de sostenibilidad.

Kimbro y sus colegas también hablan de, desarrollan, y prueban un marco con el potencial de mejorar ya sea la legitimidad de la RSE o los procesos operacionales de la RSE—o ambas-las cuales pueden resultar en respuestas positivas a las actividades de RSE. Es así como el articulo contribuye al avance de la responsabilidad social global en al menos tres maneras. Primero, el modelo se desarrolla basado en las teorías de la efectividad operacional de la RSE y medidas de rendimiento. Segundo, los hallazgos avanzan y expanden tres corrientes de investigación al integrar la literatura sobre las alianzas basadas en objetivos comunes y eficacias operacionales complementarias, estudios sobre la eficacia de la RSE y la repuesta del mercado, y teorías sobre alianzas público-privadas. Por último, este articulo ofrece un modelo replicable que las MNEs y las OSALs pueden utilizar al trabajar juntas para llevar a cabo actividades de RSE en el ámbito local e internacional, al igual que mercados emergentes y desarrollados, y para mejorar y aumentar los efectos de las actividades de la RSE que contribuyan a la justicia social, reducción de la pobreza, y un mundo más sostenible.

¿Y ahora qué? 
La última pregunta que un estudiante de MBA le hizo a cuatro conferencistas invitados a hablar sobre los problemas energéticos y la sostenibilidad global hace un par de años durante una clase, fue directa y franca: "¿Cuál es el mayor obstáculo para crear un mundo sostenible?" Cada uno de los tres primeros conferencistas rápidamente dio una respuesta elocuente y sensible basada en las oportunidades técnicas y en los problemas que se habían tratado durante la sesión. El cuarto orador, pensó un poco más, y entonces dijo algo así:

Yo considero que el mayor obstáculo para crear un mundo sostenible es que nos des-empoderamos a nosotros mismos. Hay un numero casi que infinito de cosas que podemos hacer para contribuir, pero-quizás porque no hay una singular, única bala de plata que "resuelva el problema"-o quizás porque cualquier cosa que hagamos nunca será suficiente-o por cualquier otra razón-hacemos nada. Pero si cada uno de nosotros hace algo, podremos inspirarnos los unos a los otros, y juntos podremos cambiar al mundo ... tal vez.

Quizás haya un grano de verdad en esa declaración. Como Allen Tropea-Gray deja claro en su editorial en la edición enfocada en Laudato $s i$ ' de esta revista (Tropea-Gray, 2017), hay una enorme variedad de cosas que podemos hacer como individuos y colectivamente para cuidar de nuestro hogar compartido. El desafío no es encontrar cosas que sean valiosas, emocionantes, impactantes, y personalmente gratificantes para hacer. El desafío está en estar dispuestos a decidirnos solo por una o unas pocas ... y comenzar. Como dice Chris Lowney en su libro más reciente (Lowney, 2018): "Nuestro mundo doliente esta infestado por desafíos que no pueden ser superados a menos que traigamos un corazón grande y nuestra mejor versión para enfrentar los males del mundo. Tu no pediste esa carga y responsabilidad, pero estas aquí en la cancha de juego en esto momento de la historia.... El momento te escoge a ti..." (pg. 81-82).

De pronto ahora es el momento para que cada uno de nosotros escuche a la oportunidad que nos llama con más fuerza y que sea la más atractiva en este momento ... y responder a ese llamado ... el llamado que nos puede dirigir hacia nuestra vocación.

\section{REFERENCIAS}

Brough, A., Wilkie, J., Ma, J., Isaac, M., \& Gal, D. 2016. Is eco-friendly unmanly? The green-feminine stereotype and its effect on sustainable consumption. Journal of Consumer Research, 43(4): 567-582.

Carroll, S. B. 2016. The Serengeti Rules: The quest to discover how life works and why it matters. Princeton: Princeton University Press. 
Ehrenfeld, J. 2009. Sustainability by design: A subversive strategy for transforming our consumer culture. New Haven: Yale University Press.

Francis. 2015. Laudato si': On care for our common home. Vatican City: Libreria Editrice Vaticana.

Lowney, C. 2018. Make today matter: 10 habits for a better life (and world). Chicago: Loyola Press.

Ray Anderson Foundation. (n.d.). Climbing Mount Sustainability. Available at http://www.raycandersonfoundation.org/assets/pdfs/rayslife/ EssayClimbingMountSustanability.pdf (accessed May 24, 2018).

Tropea-Gray, A. P. 2017. Pope Francis, Laudato si', and integral ecology: Perspectives on a critical issue. Journal of Management for Global Sustainability, 5(1): 1-18. 